\title{
Validación de Mediciones Retrospectivas del Consumo de Alcohol Mediante Diarios de Consumo
}

\section{Validation of Retrospective Measurements of Alcohol Consumption Through Alcohol Consumption Diaries}

\author{
Angelina Pilatti ${ }^{1,2}$, María Guadalupe Ensinck Atienza ${ }^{2}$, \\ Gabriela Rivarola Montejano ${ }^{1,2}$ y Ricardo Marcos Pautassi ${ }^{2}$ \\ ${ }^{1}$ Instituto de Investigaciones Psicológicas, \\ Consejo Nacional de Investigaciones Científicas y Técnicas, Córdoba, Argentina \\ ${ }^{2}$ Facultad de Psicología, Universidad Nacional de Córdoba
}

\begin{abstract}
Se analizó la correspondencia entre estimaciones de consumo de alcohol obtenidas mediante mediciones retrospectivas (Cuestionario de Consumo de Alcohol - CCA-) y un Diario de Consumo. Participaron 134 jóvenes de la ciudad de Córdoba, Argentina ( $M$ edad $=23,31, D E=3,18$ ) que reportaron diariamente y por 60 días su consumo de alcohol. Al final del primer y segundo mes, completaron el CCA con preguntas que medían retrospectivamente conductas de consumo de alcohol durante los 30 y 60 días previos, el Cuestionario de Identificación de Patrón Perjudicial de Consumo de Alcohol y el Cuestionario de Consecuencias del Consumo de Alcohol en Jóvenes Adultos. La recolección de datos se hizo mediante encuestas online, por lo que la muestra fue no probabilística por conveniencia. Se examinó la correlación entre las estimaciones obtenidas mediante el Diario de Consumo y aquellas obtenidas retrospectivamente (CCA). Los resultados mostraron, en general, una alta correlación entre estas estimaciones. Las correlaciones entre las estimaciones de cantidad $(\geq 0,75)$, frecuencia usual $(\geq 0,64)$ y frecuencia de consumo hasta la ebriedad $(\geq 0,74)$ tuvieron un tamaño del efecto grande, tanto para los datos del primer como del segundo mes. La mayor correspondencia se encontró para las estimaciones de cantidad usual $(0,77)$. Este estudio aporta evidencia sobre la validez de las estimaciones retrospectivas del consumo de alcohol, al mismo tiempo que se enfatiza la importancia de utilizar indicadores de consumo usual y riesgoso de alcohol en periodos temporales recientes.
\end{abstract}

Palabras clave: diario de consumo, registros retrospectivos, validación, alcohol, jóvenes

\begin{abstract}
The present study aimed to analyze the correspondence between estimates of alcohol consumption obtained through retrospective measurements (Alcohol Consumption Questionnaire-ACQ-) and an Alcohol Consumption Diary. A sample of 134 young people $(M$ age $=23.31$ years, $S D=3.18)$ from Cordoba, Argentina, reported their alcohol consumption daily over a 60-day period. At the end of the first and second month, the participants completed the $\mathrm{ACQ}$, which measures alcohol use retrospectively during the last 30 and 60 days. The participants also completed the Alcohol Use Disorders Identification Test and the Young Adult Alcohol Consequences Questionnaire. Data collection was done through online surveys, so the sample was non-probabilistic (i.e., a convenience sample). The correlation between estimates obtained through the Alcohol Consumption Diary and those obtained retrospectively (ACQ) was examined. Overall, these estimates were found to be strongly correlated. The correlations between estimates of quantity $(\geq 0.75)$, usual frequency $(\geq 0.64)$, and frequency of consumption until drunkenness $(\geq 0.74)$ had a large effect size for both the first- and second-month data. The greatest correspondence was found between estimates of usual quantity (0.77). This study provides evidence on the validity of retrospective estimates of alcohol consumption while also emphasizing the importance of using indicators of usual and hazardous alcohol consumption in recent time periods.
\end{abstract}

Keywords: consumption diary, retrospective measurements, alcohol, validation, young

El consumo de alcohol en jóvenes es un problema relevante de salud pública en casi todos los países del mundo occidental. En Argentina, por ejemplo, un estudio reciente (Pilatti et al., 2017) realizado en ingresantes universitarios indica que el 70\% reportó consumo episódico excesivo de alcohol (CEEA; consumo $\geq 56$ y 70 gramos en mujeres y hombres, respectivamente, en una misma ocasión de consumo; NIAAA Council

Angelina Pilatti (iD https://orcid.org/0000-0002-7277-0835

Gabriela Rivarola Montejano (iD https://orcid.org/0000-0003-3323-7293

Ricardo Marcos Pautassi (iD https://orcid.org/0000-0003-1876-2321

La correspondencia relativa a este artículo debe ser dirigida a Angelina Pilatti, Facultad de Psicología, Universidad Nacional de Córdoba, Bv. de la Reforma 1936, Ciudad Universitaria (5000), Córdoba, Argentina. Email: apilatti@unc.edu.ar 
approves definition of binge drinking, 2004) en los últimos seis meses; en tanto que aproximadamente un $33 \%$ reportó esta conducta de forma semanal.

La descripción y explicación del uso y abuso de alcohol deben partir de una medida válida que refleje fielmente el patrón de consumo de alcohol de los examinados. Siguiendo recomendaciones de la literatura (Dawson, 2003; Reboussin et al., 2008; Valencia Martín et al., 2014), el Cuestionario de Consumo de Alcohol (CCA; Pilatti et al., 2013) mide la frecuencia y cantidad de consumo usual y la ocurrencia y frecuencia de CEEA y consumo hasta la ebriedad. Una limitación del CCA, y de la gran mayoría de instrumentos (Hingson \& Zha, 2009; Muthén \& Muthén, 2000), es que los/las participantes informan su consumo de alcohol en función de eventos pasados. Este procedimiento podría resultar en un subrreporte del consumo (Gmel et al., 2014). Esta subestimación puede deberse a que, al estimar la media o moda de consumo, las personas examinadas no incluyen los episodios de consumo excesivo (Stahre et al., 2006). Aunque se logra una mayor precisión inquiriendo sobre periodos de referencia cortos (i.e., la última semana) o recientes (i.e., la última ocasión de consumo), esto tiene la desventaja de no reflejar el consumo usual de las personas (Dawson, 2003). El periodo de referencia más largo (i.e., el último año) supera esta limitación, pero aumenta la probabilidad de subestimaciones o fallas en la recuperación de la memoria (Dawson, 1998, 2003).

Una alternativa a la medición retrospectiva del consumo de alcohol es el uso de diarios de registro (Kiene et al., 2009; Leigh et al., 1998; Townshend \& Duka, 2002), también llamados evaluación ecológica momentánea (ecological momentary assessment; Shiffman, 2009). Estos diarios, que se basan en el registro repetido y continuo, tienen la finalidad de registrar pensamientos, sentimientos o comportamientos diversos, como conductas sexuales (Kiene et al., 2009; Leigh et al., 1998), alimenticias (Kelly \& Stephen, 2016), ciclos de sueño e insomnio (Libman et al., 2000; Short et al., 2013) y de disciplina parental (Lam et al., 2010; Peterson et al., 2002). Estos registros suelen realizarse al final del día o en momentos específicos (Shiffman, 2009). Los diarios también se han utilizado en el ámbito clínico para recolectar información, por ejemplo, sobre eventos desencadenantes del consumo de sustancias y, en función de ello, planificar tratamientos terapéuticos adecuados al contexto (Piasecki et al., 2007). Incluso, el uso de diarios ha derivado en una reducción de la conducta objetivo (e.g., consumo de alcohol) al mejorar la apreciación de cuánto se está consumiendo (Poikolainen \& Kärkkäinen, 1983).

Los diarios de registro presentan una serie de ventajas respecto de los registros retrospectivos. En primer lugar, el registro de la conducta (pensamiento o sentimiento) debe realizarse en un marco temporal cercano a su ocurrencia y, en este sentido, el reporte no está afectado por olvidos (Dawson, 2003; Piasecki et al., 2007) o sesgo telescópico (Brunner Huber et al., 2013; Leigh et al., 1998). Este último se refiere a errores en el reporte de comportamientos distantes en el tiempo: cuánto más tiempo pasa entre la conducta y el reporte de la misma más probable es que el reporte sea objeto de este tipo de sesgo que, por ejemplo, lleva a reportar una edad de inicio del consumo más cercana a la edad actual (Kuntsche et al., 2016). Los registros retrospectivos requieren una estimación del consumo típico (i.e., moda) o promedio (i.e., media) de alcohol (Dawson, 2003). Cuando el periodo de referencia es largo (e.g., un año) cualquiera de estas estimaciones es susceptible de presentar errores, especialmente cuando la conducta es poco frecuente e irregular (Dawson, 2003; Leigh et al., 1998). Los diarios de registro, en cambio, generan mediciones más ajustadas y capturan comportamientos irregulares o infrecuentes. Es por esto que, además de su uso en la clínica, resultan de utilidad para validar mediciones retrospectivas (Brunner Huber et al., 2013; Leigh et al., 1998). Los diarios de registro no están, sin embargo, exentos de desventajas. Los costos en tiempo y en dinero asociados a esta técnica de recolección de datos dificultan la obtención de muestras de gran tamaño y, especialmente, la retención de los participantes a lo largo del tiempo (Leigh et al., 1998). A diferencia de esto, el uso de mediciones retrospectivas permite medir el consumo de alcohol de una manera mucho menos costosa (en tiempo y dinero), no invasiva y más aceptable para los/as participantes, lo que hace que su uso sea casi normativo (Del Boca \& Darkes, 2003).

Hasta el momento, las estimaciones del consumo de alcohol, obtenidas mediante el CCA o mediante otro tipo de medición retrospectiva, no han sido validadas con aquellas obtenidas mediante el uso de diarios de registro. Por ello, el objetivo general de este trabajo fue validar las estimaciones del CCA, obtenidas retrospectivamente en dos periodos temporales diferentes (últimos 30 y 60 días), con estimaciones obtenidas mediante diarios de registro. En su formato clásico, los diarios incluyen una planilla donde se registra la conducta a medir (Koning et al., 2010; Peterson et al., 2002; Townshend \& Duka, 2002). Una limitación de este formato es la falta de control respecto al momento en que se completa la grilla (Leigh et al., 1998). Si la grilla se completa al final del periodo de registro, en lugar de hacerlo diariamente, se licúan las principales ventajas de los diarios de registro. Para superar estas limitaciones, se empleó una encuesta online para 
registrar diariamente la conducta de consumo de alcohol. Hasta donde se sabe, este es el primer trabajo en Latinoamérica que examinó diariamente y durante dos meses las conductas de consumo de alcohol mediante un diario de consumo.

Así, entonces, el trabajo examinó la correlación y diferencias entre las estimaciones de consumo obtenidas retrospectivamente mediante el CCA y las obtenidas mediante diarios de registro. Estas mediciones se llevaron a cabo en un periodo de 60 días. Durante dicho periodo los participantes - hombres y mujeres de entre 18 y 30 años - registraron diariamente su consumo de alcohol en un diario online y, en los días 30 y 60 , respondieron el CCA. Se utilizaron estos dos periodos temporales para examinar si una mayor ventana temporal afectaba el reporte retrospectivo. También se examinó la correlación entre las estimaciones de consumo (obtenidas mediante el CCA y los diarios de registro) con la cantidad de consecuencias negativas derivadas del uso de alcohol y el patrón perjudicial de consumo.

\section{Método}

\section{Participantes}

Se utilizó un muestreo no probabilístico por conveniencia. A través de redes sociales (i.e., Facebook, Twitter) y listas de email, se invitó a participar a jóvenes de la ciudad de Córdoba, Argentina, de entre 18 y 30 años, que hubieran consumido bebidas alcohólicas al menos dos veces al mes. La invitación indicaba que el objetivo del estudio era registrar diariamente y durante 60 días el consumo de alcohol de los participantes. Asimismo, se indicó que, entre aquellos que completaran las encuestas, se sortearían diversos premios (bijouterie, dulces, helados y entradas al cine). Las personas interesadas fueron contactadas telefónicamente para determinar el cumplimiento de los siguientes criterios de inclusión: (a) reportar no presentar afecciones psiquiátricas; (b) no estar ni haber estado bajo tratamiento para dejar de beber; (c) reportar una frecuencia de consumo de alcohol de al menos dos veces al mes. En las mujeres se descartó la posibilidad de embarazo o lactancia. Se explicó que la participación era voluntaria y se aseguró que toda la información sería tratada de manera confidencial y utilizada solo con fines estadísticos. Aunque 243 personas manifestaron su interés en participar, 109 casos fueron descartados por alguno de los siguientes motivos: no cumplir con los criterios de inclusión (34 casos), imposibilidad de ser contactados (17 casos), completaron ninguna (18 casos) o muy pocas ( 40 casos) encuestas. La muestra final quedó conformada por 134 jóvenes ( $70 \%$ mujeres; $M$ de edad $=23,31$ años, $D E=3,18)$. No se observaron diferencias en la edad en función del sexo, $t(132)=0,83, p=0,459$.

\section{Instrumentos}

\section{Diario de Consumo}

Incluyó preguntas para conocer la cantidad de vasos consumidos de una variedad de bebidas con alcohol (cerveza, vino, vino preparado, fernet, gancia [bebida consumida en Argentina], vodka, tequila, ron, ginebra, whisky), durante el día previo. Además, se incluyó una pregunta para determinar si los participantes se sintieron ebrios o intoxicados en el día previo.

\section{Cuestionario de Consumo de Alcohol (CCA)}

El consumo de alcohol fue definido como tomar un vaso o más de cualquier bebida alcohólica. Siguiendo estudios previos (Pilatti et al., 2013), se utilizaron tres preguntas:

1. Tipo de bebida alcohólica consumida habitualmente: "En los últimos 30 días, ¿cuál es la bebida que tomaste usualmente?", con opciones de respuesta como cerveza, vino/espumantes/champaña, gancia/Dr. Lemon, fernet, ron/tequila, vodka, licores [bebida alcohólica preparada a partir de destilados y saborizantes como azúcar, frutas, hierbas o especies], whisky.

2. Frecuencia de consumo de esta bebida: "¿Cada cuánto tomaste esa bebida?", con opciones de respuesta que iban desde menos de 1 vez al mes hasta 4 o más veces por semana.

3. Cantidad de vasos consumidos de esa bebida durante un mismo episodio de consumo de una ocasión típica de consumo: "¿Cuántos vasos de esa bebida tomaste en una misma ocasión?", con opciones de respuesta que iban desde 1 vaso hasta 14 vasos o más. 
Se utilizó el mismo set de tres preguntas para indagar acerca de la segunda bebida de mayor consumo. Las respuestas a la frecuencia de consumo se transformaron para determinar los días al mes de consumo de cada bebida (bebida 1 y bebida 2 ).

Las respuestas a las preguntas 1 y 3 de cada set se utilizaron para calcular la cantidad de gramos de alcohol consumidos por ocasión de consumo. Estos se calcularon sobre la base de los contenidos conocidos de alcohol de cada bebida en el mercado argentino, por ejemplo, para calcular los gramos de cerveza consumidos, se multiplicó la graduación alcohólica de esta bebida (5\%), los mililitros consumidos (un vaso contiene $250 \mathrm{ml}$ ) y la densidad del alcohol $(0,79)$, dando como resultado 9,88 gramos de alcohol por cada vaso de cerveza consumido.

Se utilizó una pregunta para determinar si, durante un mismo episodio de consumo, se consumía una única o las dos bebidas juntas. Cuando los participantes reportaron consumir las dos bebidas juntas, se sumaron los gramos de la bebida 1 y bebida 2 para determinar los gramos totales consumidos en una misma ocasión de consumo. Para calcular la cantidad de días al mes de consumo de alcohol (frecuencia total), cuando los participantes reportaron no tomar las bebidas juntas, se sumaron las respuestas a las dos preguntas de frecuencia de consumo y, cuando los participantes reportaron tomar las bebidas juntas, se utilizó la frecuencia más alta como frecuencia total.

\section{Prácticas Riesgosas de Consumo de Alcohol}

Se incluyeron, tanto en la Encuesta 1 como 2, preguntas para medir el CEEA y el consumo hasta la ebriedad. Se interrogó por la cantidad de episodios de consumo hasta la ebriedad en los 30 (Encuesta 1) y 60 (Encuesta 2) días previos y la frecuencia (desde $0=$ no en los últimos 30 [60] días hasta $6=4$ o más veces por semana) de CEEA en los 30 y 60 días previos.

\section{Versión en Español del Cuestionario de Identificación de Patrón Perjudicial de Consumo de Alcohol (Alcohol Use Disorders Identification Test, AUDIT; Rubio Valladolid et al., 1998)}

Este instrumento, uno de los más utilizados para detectar consumo problemático de alcohol (Reinert \& Allen, 2007), consta de 10 ítems con una escala ordinal de respuesta. Ocho ítems se responden con una escala de cinco puntos (desde $0=$ nunca hasta $4=$ casi a diario/diariamente), por ejemplo: "¿Con qué frecuencia tuviste remordimientos o sentimientos de culpa después de haber bebido?". Dos preguntas se responden con una escala de tres puntos $(0=n o, 2=$ sí, pero no en el último año y $4=$ sí, en el último año). Un ejemplo de estas preguntas es: "¿Has resultado herido/a o alguna otra persona ha resultado herida porque tomaste alcohol?". Una puntuación igual o mayor a 8 se utiliza como punto de corte para determinar posibles problemas con el alcohol (Loxton et al., 2008). La versión en español presenta evidencia de validez (alta especificidad [90\%] y sensibilidad [90\%]) y adecuados valores de consistencia interna, evaluada con alfa de Cronbach $(\alpha=0,77$ a $\alpha=0,86)$ en estudios previos (Pilatti, Rivarola et al., 2016; Rubio Valladolid et al., 1998). Este instrumento se completó en dos ocasiones: al final del primer mes (Encuesta 1) y del segundo mes (Encuesta 2).

\section{Versión en Español del Cuestionario de Consecuencias del Consumo de Alcohol en Jóvenes Adultos (Pilatti, Read et al., 2016)}

Se utilizó la versión validada al español en Argentina (Pilatti, Read et al., 2016) del Young Adult Alcohol Consequences Questionnaire (YAACQ; Read et al., 2006). Este instrumento mide las consecuencias del consumo de alcohol mediante 48 ítems dicotómicos $(0=n o ; 1=s \hat{\imath})$ agrupados en ocho dominios o subescalas: Social/Interpersonal (por ejemplo, "Mientras tomaba alcohol, he dicho o hecho cosas vergonzosas"), Académico/Ocupacional (por ejemplo, "La calidad de mi trabajo o estudio ha disminuido a causa de mi consumo de alcohol"), Conductas de Riesgo (por ejemplo, "He manejado un auto cuando sabía que había tomado demasiado para manejar de manera segura"), Pérdida de Control (por ejemplo, "Tomé más alcohol de lo que había planeado"), Autocuidado (por ejemplo, "No he comido de manera apropiada debido a mi consumo de alcohol"), Autopercepción (por ejemplo, "Me he sentido mal conmigo mismo por mi consumo de alcohol"), Pérdida de Consciencia (por ejemplo, "Me he sentido muy descompuesto/a o he vomitado a causa de mi consumo de alcohol") y Dependencia Fisiológica (por ejemplo, "He sentido que necesitaba tomar una bebida con alcohol al levantarme"). Los participantes debían responder si en los 30 o 60 días previos habían experimentado o no cada una de las consecuencias enumeradas en los ítems. La versión validada al español (Pilatti, Read et al., 2016) presenta adecuados índices de consistencia interna, evaluada mediante correlaciones tetracóricas, en todas las subescalas (a entre 0,70 y 0,91) y cuenta con evidencia de validez 
interna (esto es, el modelo de ocho factores correlacionados presenta excelente ajuste a los datos). Debido a la estructura de puntuación dicotómica, las respuestas afirmativas se suman, de manera tal que el puntaje total representa la cantidad de consecuencias negativas experimentadas en ese periodo. Este instrumento se completó en dos ocasiones: al final del primer y del segundo mes.

\section{Procedimiento}

Los participantes recibieron todos los días y durante un periodo de 60 días un email con un hipervínculo para completar una encuesta online con preguntas destinadas a medir su consumo de alcohol durante el día previo (Diario de Consumo). La encuesta online se implementó mediante el servidor de encuestas LimeSurvey (licencia de la Universidad Nacional de Córdoba). La primera página de cada una de las encuestas utilizadas contenía una descripción del estudio y un formulario de consentimiento informado. Siguiendo las recomendaciones de la American Psychological Association (2010), se explicitó que la participación era voluntaria, se garantizó el manejo confidencial de los datos y se incluyeron datos de contacto de los investigadores a cargo. Al final del formulario, se explicitaba que, presionando sobre la opción "siguiente", se manifestaba la conformidad a participar del estudio. Se programó la encuesta para reducir la probabilidad de datos faltantes (i.e., los participantes recibían un aviso ante respuestas faltantes). Al final del primer (30 días) y segundo (60 días) mes, los participantes completaron el CCA con preguntas destinadas a medir retrospectivamente las conductas de consumo de alcohol durante los 30 (Encuesta 1) y 60 (Encuesta 2) días previos. Estas encuestas incluyeron, además, instrumentos para medir un patrón perjudicial de consumo de alcohol (AUDIT) y consecuencias negativas del consumo de alcohol (YAACQ). Cada día, durante los 60 días del estudio, se enviaron recordatorios (vía email o telefónicamente) a quienes no habían respondido la encuesta del día previo.

\section{Análisis de Datos}

En aquellos casos que tenían al menos un 80\% de la información correspondiente a los diarios de consumo, se completaron los datos faltantes utilizando la información completa de cada participante. Puntualmente, se calculó la media del día faltante a partir de la información reportada por cada caso para ese mismo día en el resto de las semanas. Por ejemplo, si faltaba información del consumo en un día jueves, ese valor faltante fue reemplazado por la media estimada de las respuestas dadas por ese participante para los restantes jueves.

La mayoría de las variables (excepto frecuencia de consumo) presentó valores inadecuados de asimetría y curtosis $(\geq|2,0|$; George \& Mallery, 2011), indicativos de distribución no normal. Por ejemplo, la variable gramos de alcohol consumidos por ocasión presentó valores de asimetría entre 2,50 y 2,95 y curtosis entre 7,25 y 13,82. Esto motivó el uso de pruebas no paramétricas para los análisis de correlación y diferencia de medidas de tendencia central. Se utilizó el coeficiente de correlación de rangos de Spearman para determinar el grado de correspondencia entre las estimaciones obtenidas mediante el Diario de Consumo y aquellas obtenidas retrospectivamente por el CCA. A su vez, se realizaron análisis bivariados examinando la relación entre las estimaciones de consumo de alcohol obtenidas con cada registro (diario y retrospectivo) y los problemas asociados al uso de alcohol, medidos con AUDIT y YAACQ (a los 30 y 60 días, en las Encuestas 1 y 2). Se interpretó el tamaño de las asociaciones en pequeño $(0,10)$, medio $(0,30)$ y grande $(0,50 ;$ Cohen, 1988, 1992).

Las diferencias entre las estimaciones de los diferentes indicadores de consumo obtenidas con cada registro (diario y retrospectivo) se analizaron mediante el test de Wilcoxon, equivalente no paramétrico de $t$ de Student, para muestras apareadas. Específicamente, se examinaron posibles diferencias en función del método de obtención de los datos para los siguientes indicadores: (a) total de gramos de alcohol consumidos durante 30 y 60 días, (b) cantidad de episodios de consumo hasta la ebriedad durante 30 y 60 días y (c) frecuencia de CEEA durante 30 y 60 días. Para obtener el tamaño del efecto, de acuerdo con Fritz et al. (2012), se calculó una medida correlacional para datos obtenidos mediante pruebas no para métricas $[r=z / \sqrt{ } n]$ y se interpretó, de acuerdo a lo propuesto por Cohen (1988), como pequeño hasta 0,10 , medio 0,30 y grande 0,50 . Para el indicador frecuencia de consumo de alcohol, que presentó valores adecuados de asimetría y curtosis, se utilizó la prueba $t$ de Student para muestras relacionadas y, siguiendo a Cohen (1992), el tamaño del efecto se interpretó como pequeño entre 0,20 y 0,49 , mediano entre 0,50 y 0,79 y grande $\geq 0,80$.

Todos los análisis se realizaron con el programa SPSS 23.0 y se empleó un alfa de 0,05. 


\section{Resultados}

\section{Bivariados}

\section{Relación entre las Estimaciones del Consumo de Alcohol Obtenidas mediante el Diario de Consumo y aquellas Obtenidas mediante el CCA}

Las estimaciones, obtenidas mediante el Diario de Consumo, del total de gramos de alcohol consumidos durante el estudio (GD) correlacionaron directamente con aquellas obtenidas retrospectivamente (GR), con un tamaño del efecto grande, tanto con los datos del primer como del segundo mes. También se observaron correlaciones directas entre la frecuencia de consumo de alcohol, la frecuencia de CEEA y la cantidad de episodios de consumo hasta la ebriedad, estimadas mediante el diario (FD) y mediante los registros retrospectivos (FR). El tamaño del efecto de las correlaciones fue grande para ambos periodos temporales (30 o 60 días), salvo para la frecuencia de CEEA correspondiente al primer mes (con tamaño del efecto mediano), Estos resultados se presentan en la Tabla 1.

Tabla 1

Correlación entre los Distintos Indicadores de Consumo de Alcohol Estimados a Partir de los Dos Instrumentos de Medición (Diario de Consumo y CCA)

\begin{tabular}{lcccccccc}
\hline & GD & FD & CEEAD & EBD & GR & FR & CEEAR & EBR \\
\hline GD & & $0,74^{*}$ & $0,91^{*}$ & $0,57^{*}$ & $0,75^{*}$ & $0,53^{*}$ & $0,52^{*}$ & $0,51^{*}$ \\
FD & $0,74^{*}$ & & $0,56^{*}$ & $0,44^{\S}$ & $0,49^{\ddagger}$ & $0,64^{*}$ & $0,22^{\prime \prime}$ & $0,32^{\S}$ \\
CEEAD & $0,90^{*}$ & $0,55^{*}$ & & $0,60^{*}$ & $0,71^{*}$ & $0,43 \S$ & $0,53^{*}$ & $0,53^{*}$ \\
EBD & $0,59^{*}$ & $0,43^{\S}$ & $0,61^{*}$ & & $0,44^{\S}$ & $0,23^{\prime}$ & $0,27^{\prime}$ & $0,74^{*}$ \\
GR & $0,77^{*}$ & $0,52^{*}$ & $0,71^{*}$ & $0,50^{*}$ & & $0,62^{*}$ & $0,72^{*}$ & $0,49^{\S}$ \\
FR & $0,54^{*}$ & $0,74^{*}$ & $0,40^{\S}$ & $0,35^{\S}$ & $0,61^{*}$ & & $0,29^{\prime \prime}$ & $0,21^{\prime}$ \\
CEEAR & $0,52^{*}$ & $0,30^{\S}$ & $0,48^{\S}$ & $0,35^{\S}$ & $0,58^{*}$ & $0,28^{\prime \prime}$ & & $0,44^{\S}$ \\
EBR & $0,58^{*}$ & $0,32^{\S}$ & $0,55^{*}$ & $0,76^{*}$ & $0,51^{*}$ & $0,23^{\prime}$ & $0,37^{\S}$ & \\
\hline
\end{tabular}

Nota. Diagonal inferior: resultados correspondientes al primer mes; Diagonal superior: resultados correspondientes al segundo mes; todo lo terminado en D corresponde al Diario de Consumo y todo lo terminado en R corresponde al registro retropectivo (CCA); G: Gramos de alcohol consumidos; F: Frecuencia de consumo; CEEA: Consumo episódico elevado; EB: Episodios de ebriedad. . Todas las correlaciones tienen un $p \leq 0,05$. Los signos ", ${ }^{\S} \mathrm{y}^{*}$ indican un tamaño del efecto pequeño, mediano y grande, respectivamente.

\section{Relación entre las Estimaciones del Consumo de Alcohol -Obtenidas mediante el Diario de Consumo y el CCA-con las Consecuencias Negativas del Consumo (YAACQ, Encuestas 1 y 2)}

En la Tabla 2 se presenta la correlación entre los distintos indicadores de consumo de alcohol, estimados a partir del Diario de Consumo y del CCA, con la cantidad de consecuencias negativas derivadas del uso de alcohol reportadas al final del primer y del segundo mes. En general, las estimaciones obtenidas mediante el Diario de Consumo y el CCA para la cantidad de gramos de alcohol consumidos, frecuencia de consumo de alcohol, frecuencia de CEEA y cantidad de episodios de consumo hasta la ebriedad correlacionaron de manera directa con la cantidad de consecuencias negativas (i.e., puntuaciones del YAACQ). Específicamente, el tamaño del efecto fue grande para las correlaciones que involucran a las estimaciones del Diario de Consumo, excepto para la frecuencia de consumo, con un tamaño del efecto bajo en el primer mes y mediano en el segundo. En el caso de las correlaciones que involucran a las puntuaciones obtenidas mediante el CCA, se encontró un tamaño del efecto grande con la estimación de ebriedad, mientras que las correlaciones con el resto de los indicadores tuvieron, en general, un tamaño del efecto mediano. Solo para la frecuencia usual, medida retrospectivamente, no se encontró correlación. 
Tabla 2

Correlación entre los Distintos Indicadores de Consumo de Alcohol, Estimados a Partir del Diario de Consumo y del CCA, con las Puntuaciones del YAACQ y el AUDIT

\begin{tabular}{lcccc}
\hline & YAACQ 30 & YAACQ 60 & AUDIT 30 & AUDIT 60 \\
\hline GD & $0,50^{*}$ & $0,55^{*}$ & $0,62^{*}$ & $0,70^{*}$ \\
GR & $0,46^{\S}$ & $0,45^{\S}$ & $0,62^{*}$ & $0,63^{*}$ \\
FD & $0,23^{*}$ & $0,38^{\S}$ & $0,36^{\S}$ & $0,50^{*}$ \\
FR & 0,15 & $0,25^{\prime}$ & $0,31^{\S}$ & $0,36^{\S}$ \\
CEEAD & $0,54^{*}$ & $0,55^{*}$ & $0,59^{*}$ & $0,65^{*}$ \\
CEEAR & $0,43^{\S}$ & $0,40^{\S}$ & $0,49^{\S}$ & $0,55^{*}$ \\
EBD & $0,55^{*}$ & $0,63^{*}$ & $0,58^{*}$ & $0,54^{*}$ \\
EBR & $0,55^{*}$ & $0,61^{*}$ & $0,61^{*}$ & $0,58^{*}$ \\
\hline
\end{tabular}

Nota. Todo lo terminado en D corresponde al Diario de Consumo y todo lo terminado en $\mathrm{R}$ corresponde al registro retrospectivo (CCA); G: gramos de alcohol consumidos; F: Frecuencia de consumo; CEEA: Consumo episódico elevado; EB: episodios de ebriedad; 30/60: 30/60 días; YAACQ: cuestionario de consecuencias negativas del consumo de alcohol; AUDIT: instrumento de detección de patrones perjudiciales de consumo de alcohol. Todas las correlaciones tienen

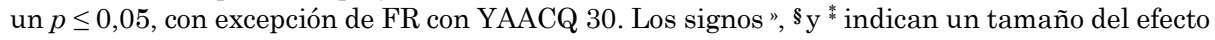
pequeño, mediano y grande, respectivamente.

\section{Relación entre las Estimaciones del Consumo de Alcohol -Obtenidas Mediante el Diario de Consumo y el CCA-con las Puntuaciones del AUDIT (Encuestas 1 y 2)}

Las correlaciones entre los distintos indicadores de consumo de alcohol, estimados a partir del Diario de Consumo y del CCA, con las puntuaciones del AUDIT fueron directas. El tamaño del efecto fue, en general, grande para todas las correlaciones que incluían las estimaciones obtenidas a partir del Diario de Consumo, excepto para la frecuencia usual del primer mes, que tuvo un tamaño mediano. Respecto de las correlaciones que incluían a las mediciones obtenidas a partir del CCA, a excepción de la frecuencia usual (del primer y segundo mes) y del CEEA en el primer mes, que tuvieron efecto mediano, las demás correlaciones tuvieron un tamaño del efecto grande. Ver estos resultados en la Tabla 2.

\section{Diferencias en Función del Método de Estimación}

A continuación, se presentan los resultados al examinar diferencias en las estimaciones del consumo de alcohol en función del método de medición (i.e., diario versus registro retrospectivo).

\section{Cantidad de Gramos de Alcohol por Ocasión de Consumo}

La prueba de Wilcoxon arrojó diferencias en la cantidad de gramos de alcohol consumidos en función del método utilizado. Específicamente, tanto para el primer mes, $Z=-2,90, p=0,004$, como para el segundo mes, $Z=-2,53, p=0,011$, la cantidad de gramos de alcohol reportada a partir del Diario de Consumo fue más alta que la cantidad reportada mediante el CCA. El tamaño del efecto de la diferencia fue pequeño, tanto para el primer mes $(r=0,26)$ como para el segundo $(r=0,25)$. Estos resultados se presentan en el panel izquierdo de la Figura 1.

\section{Frecuencia de Consumo de Alcohol}

Se encontraron diferencias en la frecuencia de consumo de alcohol (i.e., días de consumo) en función del método de estimación, tanto en el primer mes, $t(120)=6,25, p<0,001$, como en el segundo, $t(104)=7,34$, $p<0,001$. En ambos casos, la frecuencia de consumo de alcohol reportada mediante el Diario de Consumo fue mayor que la reportada retrospectivamente mediante el CCA. El tamaño del efecto de la diferencia fue pequeño para las comparaciones en el primer mes $(d=0,40)$ y mediano para el segundo mes $(d=0,60)$. Estos resultados se presentan en el panel derecho de la Figura 1. 


\section{Frecuencia de CEAA}

Se encontraron diferencias en la frecuencia de CEEA en función del método utilizado, tanto en el primer mes, $Z=-3,24, p<0,001$, como en el segundo, $Z=-4,47, p<0,001$. En ambos casos, la frecuencia de CEEA reportada mediante el Diario de Consumo fue mayor que la reportada retrospectivamente. El tamaño del efecto de esta diferencia fue pequeño para el primer mes $(r=0,29)$ y mediano para el segundo mes $(r=0,44)$ Estos resultados se presentan en el panel izquierdo de la Figura 2.

\section{Cantidad de Episodios de Consumo hasta la Ebriedad}

Tanto para el primer mes, $Z=-4,62, p<0,001$, como para el segundo, $Z=-5,72, p<0,001$, la cantidad de episodios de consumo hasta la ebriedad reportada mediante el Diario de Consumo fue mayor que la cantidad de estos episodios reportada mediante el CCA. El tamaño del efecto de la diferencia fue mediano para el primer mes $(r=0,42)$ y grande para el segundo mes $(r=0,56)$. Estos resultados se presentan en el panel derecho de la Figura 2.

\section{Discusión}

La medición del consumo de alcohol mediante el uso de registros diarios se ha visto limitada por los costos de tiempo y dinero asociados a este método. Sin embargo, los registros diarios son muy útiles para, entre otras cosas, validar estimaciones obtenidas mediante registros retrospectivos (Brunner Huber et al., 2013; Leigh et al., 1998). De manera similar a estudios previos (Hilton, 1989; Koning et al., 2010), los resultados de este estudio muestran una moderada a alta correspondencia entre el consumo de alcohol reportado a través del Diario de Consumo y el reportado retrospectivamente mediante el CCA. Esto se observó para una variedad de indicadores, tanto de consumo usual como de prácticas riesgosas de consumo de alcohol, como el consumo hasta la ebriedad. Esta alta correlación entre medidas de cantidad y frecuencia usual ya había sido señalada por un estudio previo (Koning et al., 2010), en el que las asociaciones entre ambos tipos de medidas fueron $\geq 0,60$, aunque en población adolescente. Si bien la correlación entre las estimaciones de la cantidad de gramos de alcohol fue similar para los 30 y 60 días, se observó una reducción en el tamaño de la correlación para la frecuencia de consumo correspondiente al segundo mes. Esto sugiere que, al menos para la frecuencia usual de consumo de alcohol, un periodo más acotado y cercano en el tiempo permite reportes de mayor consistencia que aquellos que involucran un lapso temporal más grande.

Este efecto temporal no parece haberse expresado de la misma manera para la frecuencia de una práctica riesgosa de consumo de alcohol, como es el consumo hasta la ebriedad. Las correlaciones entre la frecuencia de esta práctica, reportada mediante el diario y mediante el CCA, tuvieron tamaños del efecto similares para el primer y para el segundo mes. Nótese, sin embargo, que más allá de la estabilidad temporal entre estas correlaciones, la correspondencia entre la frecuencia de CEEA (otro tipo de práctica riesgosa) reportada mediante el diario y la reportada retrospectivamente, fue la más baja entre pares de mismos indicadores, incluso con un tamaño del efecto mediano para el primer mes y grande para el segundo mes. Por un lado, es posible que las prácticas menos frecuentes o con elementos distintivos particulares, como el consumo hasta la ebriedad, sean menos sensibles a sesgos por olvido. Esto podría subyacer a la estabilidad temporal hallada en las correlaciones. Por otro lado, la menor correspondencia observada para la frecuencia de CEEA sugiere que el reporte de este tipo de práctica está sujeto a variaciones según el método de registro que se utilice. Una posibilidad es que, retrospectivamente, se pasen por alto algunos de estos episodios de consumo excesivo. Estas inconsistencias en el reporte de CEEA a lo largo del tiempo se han puesto de manifiesto en estudios longitudinales (Schulenberg et al., 1996; Weingardt et al., 1998). Este reporte podría ser especialmente inconsistente ante la ausencia de elementos o consecuencias particulares como una descompostura o intoxicación mayor del estado de ebriedad.

Algunos resultados sugieren un subrreporte del consumo en las estimaciones obtenidas mediante registros retrospectivos. Específicamente, la frecuencia (usual o de prácticas riesgosas) y la cantidad de consumo de alcohol reportada mediante los registros diarios son significativamente más altaa que cuando los mismos indicadores se reportan de manera retrospectiva. Estas diferencias fueron de mayor tamaño para el lapso de 60 días que para el periodo temporal de 30 días, lo que sugiere que la precisión o correspondencia del reporte retrospectivo disminuye cuando aumenta el periodo temporal o cuando el periodo de referencia se aleja más del momento del reporte (Dawson, 2003). 
Figura 1

Cantidad de Alcohol Consumido y Frecuencia de Consumo Estimadas a Partir del Registro Diario y Retrospectivo

Cantidad de alcohol consumido

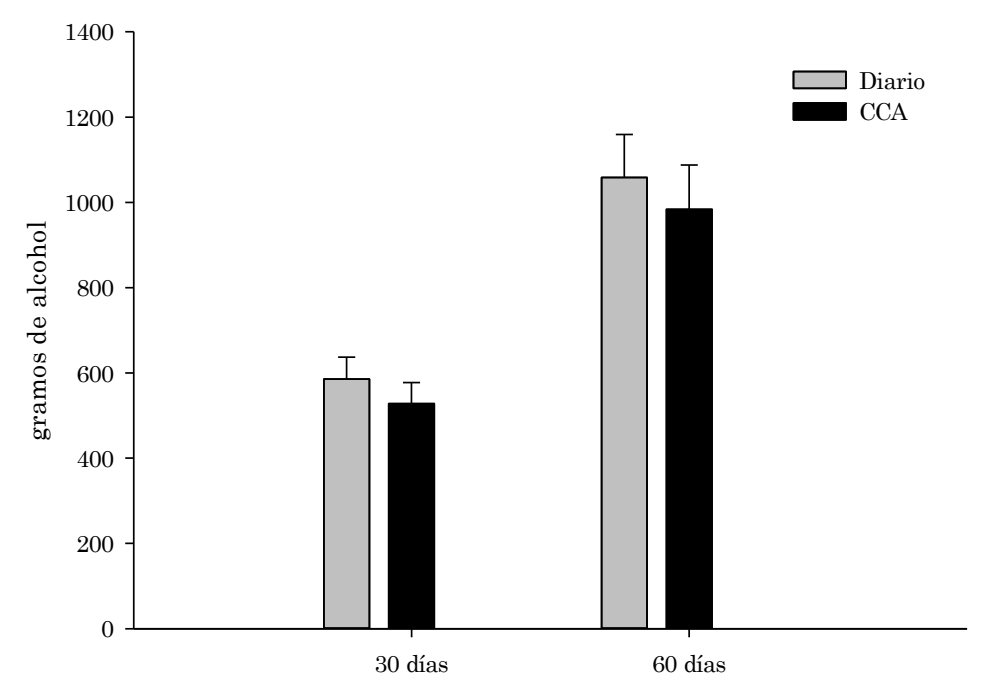

Frecuencia de consumo de alcohol

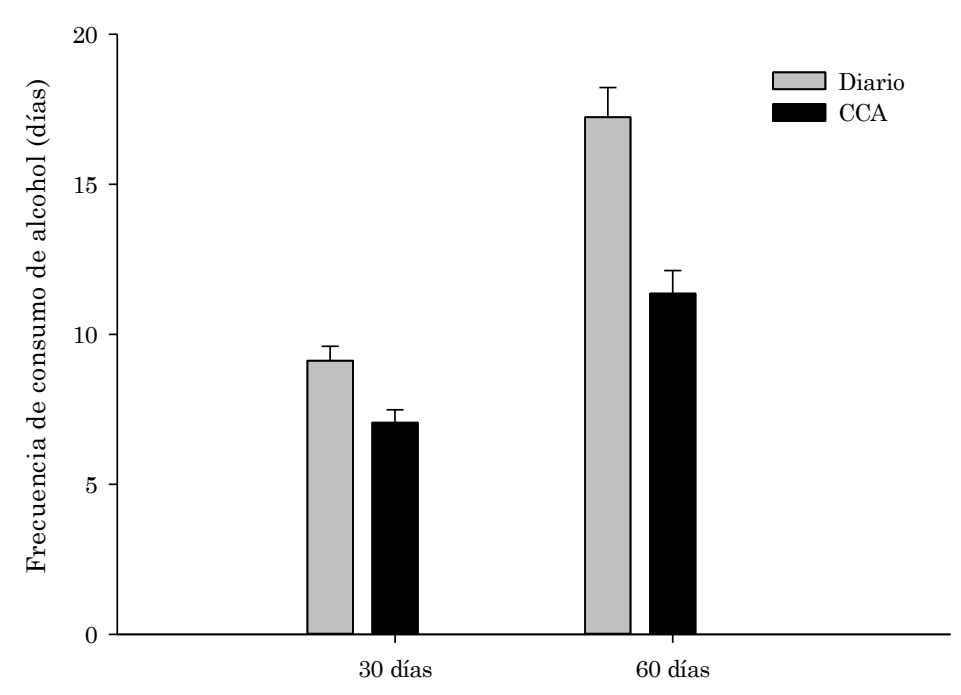

Nota. En el panel izquierdo se muestran los gramos de alcohol por ocasión de consumo y en el panel derecho, la frecuencia de consumo. Los resultados corresponden a los 30 y 60 días del estudio. Los datos se presentan como medias y las líneas verticales corresponden al error estándar de la media. 


\section{Figura 2}

Consumo Episódico Elevado de Alcohol y Consumo hasta la Ebriedad Estimadas a Partir del Registro Diario y Retrospectivo

Frecuencia de consumo de alcohol

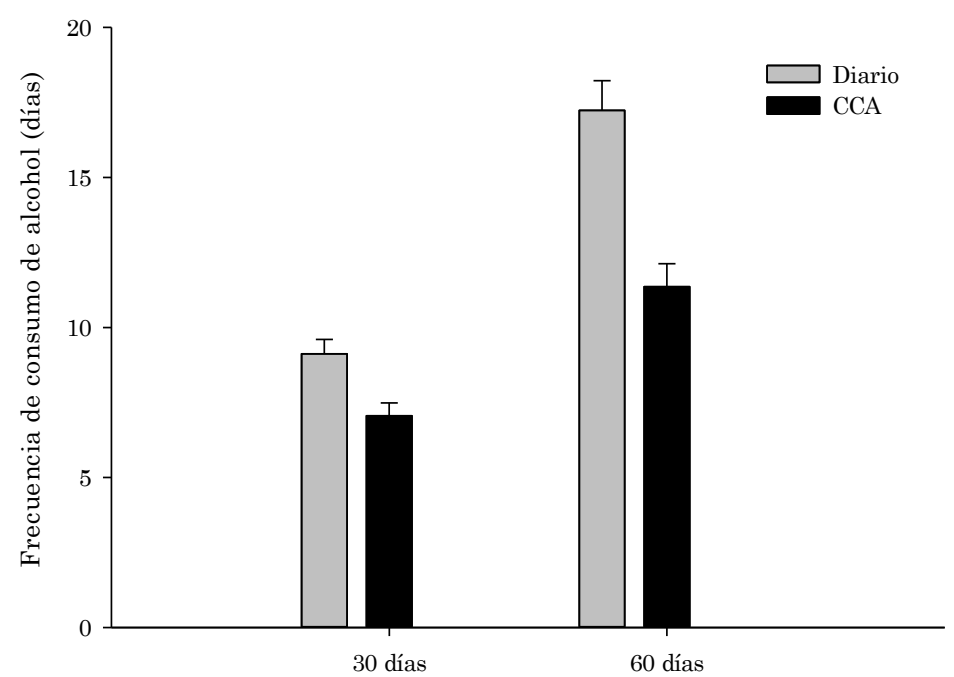

Frecuencia de episodios de ebriedad

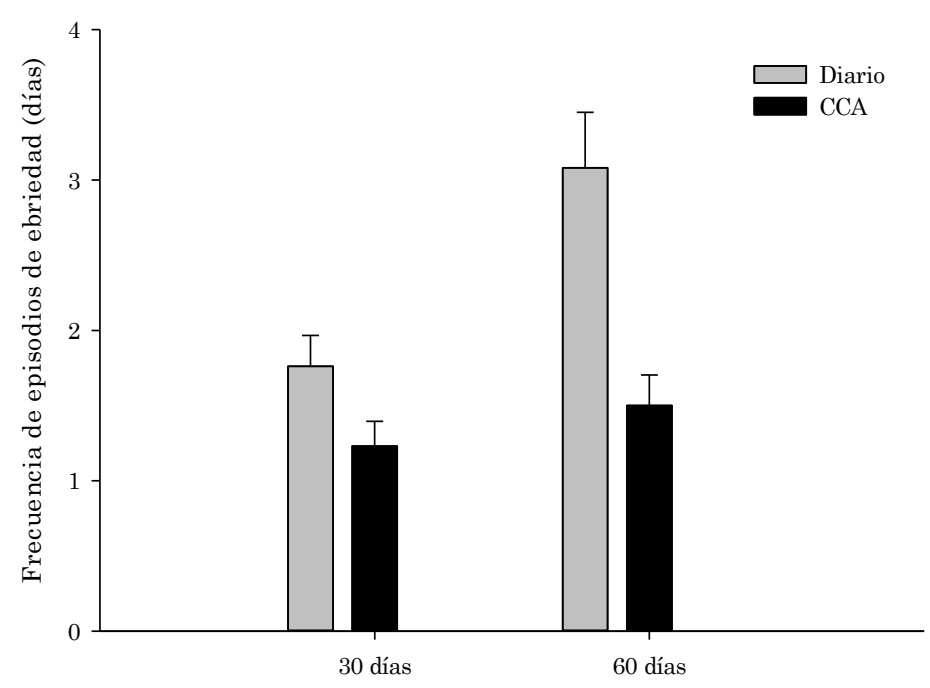

Nota. En el panel izquierdo se muestra la frecuencia de consumo episódico elevado de alcohol (CEEA) y en el panel derecho, la frecuencia de consumo hasta la ebriedad. Los resultados corresponden a los 30 y 60 días del estudio. Los datos se presentan como medias y las líneas verticales corresponden al error estándar de la media. 
En síntesis, los resultados del presente trabajo sugieren que, a pesar del subrreporte del consumo en las estimaciones retrospectivas, hay una correspondencia aceptable entre las estimaciones del consumo de alcohol obtenidas mediante el registro diario de esta conducta y aquellas obtenidas mediante registros retrospectivos. Esta consistencia es más marcada cuando el periodo temporal es reciente (un mes), disminuyendo para un periodo temporal más extenso (dos meses). En base a esto, se sugiere a los investigadores que usen registros de tipo retrospectivo de periodos temporales cortos y cercanos en el tiempo.

La evidencia previa indica que solicitar a los participantes que reporten la media de su consumo usual lleva, usualmente, a una subestimación del consumo. Esto probablemente obedece a la falta de consideración de las ocasiones de consumo excesivo o riesgoso (Stahre et al., 2006). También debe notarse que realizar dicho cálculo puede implicar desafíos cognitivos, los cuales se solapan con aquellos que resultan afectados por el consumo crónico de alcohol. De acuerdo con los resultados del presente trabajo, este sesgo podría evitarse utilizando preguntas que interroguen sobre modalidades de consumo riesgoso, como el CEEA y el consumo hasta la ebriedad.

Este trabajo no está exento de limitaciones. Participaron del estudio jóvenes que reportaron haber consumido alcohol al menos dos veces en el mes previo. La lógica subyacente a este criterio de inclusión es que los objetivos del estudio implicaban correlación entre conductas de consumo de alcohol, por lo que era necesario contar con participantes bebedores regulares de alcohol. Más allá de esto, sería valioso explorar las relaciones aquí examinadas en una muestra con una adecuada representación de diferentes patrones o modalidades de consumo de alcohol que involucren diferente cantidad (baja, moderada, alta) y frecuencia (infrecuente, frecuente). La muestra, asimismo, estuvo conformada por un mayor número de mujeres que de hombres, por lo que también sería útil avanzar sobre estos objetivos en una muestra con una adecuada representación en función del sexo. Otro aspecto a tener en cuenta es que la precisión del reporte retrospectivo pudo haber estado influida por el registro diario. Es posible y esperable que registrar diariamente las conductas de consumo haya aumentado el recuerdo al momento de reportar retrospectivamente estas conductas. La evidencia previa indica, sin embargo, que este efecto es mínimo (Glovannucci et al., 1991) o inexistente (Pietinen et al., 1988).

Otra limitación es el solapamiento en los registros retrospectivos del consumo referidos a los 30 y los 60 días anteriores. Aunque lo usual es evitar este tipo solapamiento, en este trabajo fue necesario hacerlo para examinar si variaciones en la ventana temporal del registro retrospectivo alteraba o influía sobre el reporte. Finalmente, el método utilizado para calcular los gramos de alcohol consumidos dificulta la comparación entre distintas bebidas, por lo que en los últimos años se ha popularizado el uso de cuestionarios que utilizan una imagen que describe el volumen (en mililitros) de diferentes bebidas alcohólicas equivalentes a una unidad estándar. Sin embargo, el uso de la unidad estándar tampoco está exento de limitaciones (National Institute on Alcohol Abuse and Alcoholism, 2005).

Más allá de estas limitaciones, este trabajo realiza importantes aportes y entrega información de interés a la hora de planificar mediciones retrospectivas del consumo de alcohol.

\section{Referencias}

American Psychological Association. (2010). Publication manual of the American Psychological Association (6 ${ }^{\mathrm{a}}$ ed.).

Brunner Huber, L. R., Broel, E. C., Mitchelides, A. N., Dmochowski, J., Dulin, M. \& Scholes, D. (2013). Comparison of prospective daily diaries and retrospective recall to measure oral contraceptive adherence. Contraception, 88, 492-497. https://doi.org/10.1016/j.contraception.2013.02.007

Cohen, J. (1988). Statistical power analysis for the behavioral sciences (2a ed.). Lawrence Erlbaum.

Cohen, J. (1992). A power primer. Psychological Bulletin, 112, 155-159. https://doi.org/10.1037//0033-2909.112.1.155

Dawson, D. A. (1998). Measuring alcohol consumption: Limitations and prospects for improvement. Addiction, 93(7), 965-968. https://doi.org/10.1080/09652149835143

Dawson, D. A. (2003). Methodological issues in measuring alcohol use. Alcohol Research \& Health, 27(1), 18-29. https://www.ncbi.nlm.nih.gov/pmc/articles/PMC6676704/

Del Boca, F. K. \& Darkes, J. (2003). The validity of self-reports of alcohol consumption: State of the science and challenges for research. Addiction, 98(s2), 1-12. https://doi.org/10.1046/j.1359-6357.2003.00586.x

Fritz, C. O., Morris, P. E. \& Richler, J. J. (2012). Effect size estimates: Current use, calculations, and interpretation. Journal of Experimental Psychology: General, 141(1), 2-18. https://doi.org/10.1037/a0024338

George, D. \& Mallery, P. (2011). SPSS for Windows step by step: A simple guide and reference 18.0 update (11 a ed.). Allyn \& Bacon.

Glovannucci, E., Colditz, G., Stampfer, M. J., Rimm, E. B., Litin, L., Sampson, L. \& Willett, W. C. (1991). The assessment of alcohol consumption by a simple self-administered questionnaire. American Journal of Epidemiology, 133(8), 810-817. https://doi.org/10.1093/oxfordjournals.aje.a115960

Gmel, G., Studer, J., Deline, S., Baggio, S., N'Goran, A., Mohler-Kuo, M. \& Daeppen, J. -B. (2014). More is not always better comparison of three instruments measuring volume of drinking in a sample of young men and their association with consequences. 
Journal of Studies on Alcohol and Drugs, 75(5), 880-888. https://doi.org/10.15288/jsad.2014.75.880

Hilton, M. E. (1989). A comparison of a prospective diary and two summary recall techniques for recording alcohol consumption. British Journal of Addiction, 84(9), 1085-1092. https://doi.org/10.1111/j.1360-0443.1989.tb00792.x

Hingson, R. W. \& Zha, W. (2009). Age of drinking onset, alcohol use disorders, frequent heavy drinking, and unintentionally injuring oneself and others after drinking. Pediatrics, 123(6), 1477-1484. https://doi.org/10.1542/peds.2008-2176

Kelly, A. C. \& Stephen, E. (2016). A daily diary study of self-compassion, body image, and eating behavior in female college students. Body Image, 17, 152-160. https://doi.org/10.1016/j.bodyim.2016.03.006

Kiene, S. M., Barta, W. D., Tennen, H. \& Armeli, S. (2009). Alcohol, helping young adults to have unprotected sex with casual partners: Findings from a daily diary study of alcohol use and sexual behavior. Journal of Adolescent Health, 44(1), 73-80. https://doi.org/10.1016/j.jadohealth.2008.05.008

Koning, I. M., Harakeh, Z., Engels, R. C. M. E. \& Vollebergh, W. A. M. (2010). A comparison of self-reported alcohol use measures by early adolescents: Questionnaires versus diary. Journal of Substance Use, 15(3), 166-173. https://doi.org/10.3109/14659890903013091

Kuntsche, E., Rossow, I., Engels, R. \& Kuntsche, S. (2016). Is 'age at first drink' a useful concept in alcohol research and prevention? We doubt that. Addiction, 111(6), 957-965. https://doi.org/10.1111/add.12980

Lam, J., Barr, R. G., Catherine, N., Tsui, H., Hahnhaussen, C. L., Pauwels, J. \& Brant, R. (2010). Electronic and paper diary recording of infant and caregiver behaviors. Journal of Developmental \& Behavioral Pediatrics, 31(9), 685-693. https://doi.org/10.1097/DBP.0b013e3181e416ae

Leigh, B. C, Guillmore, M. R. \& Morrison, D. M. (1998). Comparison of diary and retrospective measures for recording alcohol consumption and sexual activity. Journal of Clinical Epidemiology, 51(2), 119-127. https://doi.org/10.1016/S0895-4356(97)00262-X

Libman, E., Fichten, C. S., Bailes, S. \& Amsel, R. (2000). Sleep questionnaire versus sleep diary: Which measure is better? International Journal of Rehabilitation and Health, 5(3), 205-209. https://doi.org/10.1023/A:1012955423123

Loxton, N. J., Nguyen, D., Casey, L. \& Dawe, S. (2008). Reward drive, rash impulsivity and punishment sensitivity in problems gamblers. Personality and Individual Differences, 45(2), 167-173. https://doi.org/10.1016/j.paid.2008.03.017

Muthén, B. O. \& Muthén, L. K. (2000). The development of heavy drinking and alcohol-related problems from ages 18 to 37 in a U.S. national sample. Journal of Studies on Alcohol and Drugs, 61(2), 290-300. https://doi.org/10.15288/jsa.2000.61.290

National Institute on Alcohol Abuse and Alcoholism. (2005). ¿Qué es una bebida estándar? Recuperado el 14 de Diciembre de 2018 de https://pubs.niaaa.nih.gov/publications/Practitioner/PocketGuide/Spanish/pocket_guide2_sp.htm

NIAAA Council approves definition of binge drinking. (2004, Febrero 5). NIAAA Newsletter, Winter(3), 3. http://pubs.niaaa.nih.gov/publications/Newsletter/winter2004/Newsletter Number3.pdf

Peterson, L., Tremblay, G., Ewigman, B. \& Popkey, C. (2002). The Parental Daily Diary: A sensitive measure of the process of change in a child maltreatment prevention program. Behavior Modification, 26(5), 627-647. https://doi.org/10.1177/014544502236654

Piasecki, T. M., Hufford, M. R., Solhan, M. \& Trull, T. J. (2007). Assessing clients in their natural environments with electronic diaries: Rationale, benefits, limitations, and barriers. Psychological Assessment, 19(1), 25-43. https://doi.org/10.1037/1040-3590.19.1.25

Pietinen, P., Hartman, A. M., Haapa, E., Räsänen, L., Haapakoski, J., Palmgren, J., Albanes, D., Virtamo, J. \& Huttunen, J. K. (1988). Reproducibility and validity of dietary assessment instruments: I. A self-administered food use questionnaire with a portion size picture booklet. American Journal of Epidemiology, 128(3), 655-666. https://doi.org/10.1093/oxfordjournals.aje.a115013

Pilatti, A., Godoy, J. C., Brussino, S. A. \& Pautassi, R. M. (2013). Patterns of substance use among Argentinean adolescents and analysis of the effect of age at first alcohol use on substance use behaviors. Addictive Behaviors, 38(12), 2847-2850. https://doi.org/10.1016/j.addbeh.2013.08.007

Pilatti, A., Read, J. P. \& Caneto, F. (2016). Validation of the Spanish version of the Young Adult Alcohol Consequences Questionnaire (S-YAACQ). Psychological Assessment, 28(5), e49-e61. https://doi.org/10.1037/pas0000140

Pilatti, A., Read, J. P. \& Pautassi, R. M. (2017). ELSA 2016 cohort: Alcohol, tobacco, and marijuana use and their association with age of drug use onset, risk perception, and social norms in Argentinean college freshmen. Frontiers in Psychology, 8, Artículo 1452. https://doi.org/10.3389/fpsyg.2017.01452

Pilatti, A., Rivarola Montejano, G., Lozano, O. M. \& Pautassi, R. M. (2016). Relación entre impulsividad y consumo de alcohol en hombres y mujeres argentinos. Quaderns de Psicologia, 18(1), 75-91. https://doi.org/10.5565/rev/apsicologia.1329

Poikolainen, K. \& Kärkkäinen, P. (1983). Diary gives more accurate information about alcohol consumption than questionnaire. Drug and Alcohol Dependence, 11(2), 209-216. https://doi.org/10.1016/0376-8716(83)90080-7

Read, J. P., Kahler, C. W., Strong, D. R. \& Colder, C. R. (2006). Development and preliminary validation of the Young Adult Alcohol Consequences Questionnaire. Journal of Studies on Alcohol and Drugs, 67(1), 169-177. https://doi.org/10.15288/jsa.2006.67.169

Reboussin, B. A., Ip, E. H. \& Wolfson, M. (2008). Locally dependent latent class models with covariates: An application to under-age drinking in the USA. Journal of the Royal Statistical Society: Series A, 171(4), 877-897. https://doi.org/10.1111/j.1467-985X.2008.00544.x

Reinert, D. F. \& Allen, J. P. (2007). The Alcohol Use Disorders Identification Test: An update of research findings. Alcoholism: Clinical \& Experimental Research, 31(2), 185-199. https://doi.org/10.1111/j.1530-0277.2006.00295.x

Rubio Valladolid, G., Bermejo Vicedo, J., Caballero Sánchez-Serrano, M. C. \& Santo-Domingo Carrasco, J. (1998). Validación de la prueba para la identificación de trastornos por uso de alcohol (AUDIT) en atención primaria. Revista Clínica Española, 198(1), 1114. https://pubmed.ncbi.nlm.nih.gov/9534342/

Schulenberg, J., O'Malley, P. M., Bachman, J. G., Wadsworth, K. N. \& Johnston, L. D. (1996). Getting drunk and growing up: Trajectories of frequent binge drinking during the transition to young adulthood. Journal of Studies on Alcohol and Drugs, 57(3), 289-304. https://doi.org/10.15288/jsa.1996.57.289

Shiffman, S. (2009). Ecological momentary assessment (EMA) in studies of substance use. Psychological Assessment, $21(4), 486$. https://doi.org/10.1037/a0017074

Short, M. A., Gradisar, M., Lack, L. C., Wright, H. R. \& Chatburn, A. (2013). Estimating adolescent sleep patterns: Parent reports versus adolescent self-report surveys, sleep diaries, and actigraphy. Nature and Science of Sleep, 5, 23-26. https://doi.org/10.2147/NSS.S38369

Stahre, M., Naimi, T., Brewer, R. \& Holt, J. (2006). Measuring average alcohol consumption: The impact of including binge drinks in quantity-frequency calculations. Addiction, 101(12), 1711-1718. https://doi.org/10.1111/j.1360-0443.2006.01615.x

Townshend, J. M. \& Duka, T. (2002). Patterns of alcohol drinking in a population of young social drinkers: A comparison of questionnaire and diary measures. Alcohol and Alcoholism, 37(2), 187-192. https://doi.org/10.1093/alcalc/37.2.187

Valencia Martín, J. L., González, M. J. \& Galán, I. (2014). Aspectos metodológicos en la medición del consumo de alcohol: la importancia de los patrones de consumo. Revista Española de Salud Pública, 88(4), 433-446. https://doi.org/10.4321/S1135-57272014000400002 
Weingardt, K. R., Baer, J. S., Kivlahan, D. R., Roberts, L. J., Miller, E. T. \& Marlatt, G. A. (1998). Episodic heavy drinking among college students: Methodological issues and longitudinal perspectives. Psychology of Addictive Behaviors, 12(3), 155-167. https://doi.org/10.1037/0893-164X.12.3.155

Fecha de recepción: Febrero de 2019.

Fecha de aceptación: Junio de 2020. 